\title{
Results of the astrometry and direct imaging testbed for exoplanet detection
}

Eduardo A. Bendek, Ruslan Belikov, Eugene Pluzhnik, Olivier Guyon, Thomas Milster, et al.

Eduardo A. Bendek, Ruslan Belikov, Eugene Pluzhnik, Olivier Guyon, Thomas Milster, Lee Johnson, Emily Finan, Justin Knight, Alexander Rodack, "Results of the astrometry and direct imaging testbed for exoplanet detection," Proc. SPIE 10400, Techniques and Instrumentation for Detection of Exoplanets VIII, 104001G (1 September 2017); doi: 10.1117/12.2274729

EDent: SPIE Optical Engineering + Applications, 2017, San Diego, California, United States 


\title{
Results of the astrometry and direct imaging testbed for exoplanet detection
}

\author{
Eduardo A. Bendek ${ }^{\mathrm{c}}$, Ruslan Belikov ${ }^{\mathrm{c}}$, Eugene Pluzhnik ${ }^{\mathrm{c}}$, Olivier Guyon ${ }^{\mathrm{a}}$, Thomas Milster ${ }^{\mathrm{d}}$, Lee \\ Johnson $^{\mathrm{d}}$, Emily Finan ${ }^{\mathrm{d}}$, Justin Knight ${ }^{\mathrm{d}}$, and Alexander Rodack ${ }^{\mathrm{d}}$ \\ ${ }^{a}$ University of Arizona, Steward Observatory, 933 N Cherry Ave, Tucson, AZ, USA 85721; \\ ${ }^{\mathrm{b}}$ Lawrence Livermore National Laboratory, 7000 East Ave, Livermore, CA 94550; \\ 'NASA Ames Research Center, Moffett Field, CA 94035, USA \\ ${ }^{d}$ University of Arizona, College of Optical Sciences, 1630 E. University Ave, Tucson, AZ, USA \\ 85721;
}

\begin{abstract}
Measuring masses of long-period planets around F, G, and K stars is necessary to characterize exoplanets and assess their habitability. Imaging stellar astrometry offers a unique opportunity to solve radial velocity system inclination ambiguity and determine exoplanet masses. The main limiting factor in sparse-field astrometry, besides photon noise, is the non-systematic dynamic distortions that arise from perturbations in the optical train. Even space optics suffer from dynamic distortions in the optical system at the sub- $\mu$ as level. To overcome this limitation we propose a diffractive pupil that uses an array of dots on the primary mirror creating polychromatic diffraction spikes in the focal plane, which are used to calibrate the distortions in the optical system. By combining this technology with a high-performance coronagraph, measurements of planetary systems orbits and masses can be obtained faster and more accurately than by applying traditional techniques separately. In this paper, we present the results of the combined astrometry and and highcontrast imaging experiments performed at NASA Ames Research Center as part of a Technology Development for Exoplanet Missions program. We demonstrated $2.38 \times 10^{-5} \lambda / \mathrm{D}$ astrometric accuracy per axis and $1.72 \times 10^{-7}$ raw contrast from 1.6 to $4.5 \lambda / \mathrm{D}$. In addition, using a simple average subtraction post-processing we demonstrated no contamination of the coronagraph field down to $4.79 \times 10^{-9}$ raw contrast.
\end{abstract}

Keywords: Distortion, diffractive pupil, high-precision astrometry, direct imaging, exoplanet detection, planet masses

\section{INTRODUCTION}

Astrometry is one of the most promising exoplanet detection and characterization techniques, allowing the determination of planetary mass and orbit, solving the system inclination ambiguity, and determining coplanarity of planetary systems [1]. Moreover, high-precision astrometry enhances other exoplanet detection techniques, such as direct imaging. Direct imaging is required to study the exoplanet atmosphere composition through spectroscopy, while astrometry is required to measure exoplanet mass. Pairing these techniques together characterizes an exoplanet in greater detail than using them separately, as the combined measurements offer greater detection sensitivity and reliability [2,3,4]. However, only a few planets have been detected using astrometry because the astrometric signal of most exoplanets, including any Earth-like planet around nearby stars $(<10 \mathrm{pc})$, is on the order of sub- $\mu$ as and is therefore undetectable for today's most advanced instrumentation.

\subsection{COMBINED ASTROMETRY AND DIRECT IMAGING}

The main limiting factor in sparse-field astrometry, besides photon noise, is the non-systematic dynamic distortions that arise from perturbations in the optical train $[5,6]$. To improve the astrometric measurements, it is necessary to increase

Techniques and Instrumentation for Detection of Exoplanets VIII, edited by Stuart Shaklan, Proc. of SPIE Vol. 10400, 104001G · C 2017 SPIE · CCC code: 0277-786X/17/\$18 · doi: 10.1117/12.2274729 
the number of background stars, which is achieved by using a wider-field camera. However, as the Field of View (FoV) increases, distortion dominates the error budget; hence, the challenge is to achieve long-term distortion stability on a wide-field camera.

Even space optics suffer from dynamic distortions in the optical system at the sub- $\mu$ as level. To overcome this limitation, a concept has been proposed [5] which uses a Diffractive Pupil (DP) to generate precise fiducial features in the image plane, which appear as radial streaks or spikes. These diffractive features can calibrate dynamic or relative distortions because they are imaged by the same optical system "as the target star". Therefore, their positions also change with distortions, thus serving as a reference for calibration. Other researchers have proposed using a diffractive grid for narrow-field astrometry applications $[7,8]$. Here we propose the application of the DP concept to wide-field cameras. The astrometric precision should improve rapidly with the $\mathrm{FoV}$ as the number of background stars increases.

To create diffractive fiducial features in the image plane, a periodic array of small dots is imprinted on the primary mirror of the telescope. These dots diffract the incident light and generate a new array of dots with inverse spatial frequency, as predicted by Fraunhofer far-field diffraction. These dots are smeared radially in broadband light forming "diffraction spikes".

The astrometric signal of the central star is then measured by comparing the position of the diffraction spikes to the background stars instead of comparing directly to the host star point spread function (PSF). In the presence of dynamic distortions between measurements, as shown in Fig. 1.1, the pixel location of a star is biased by the distortions. However, if the position of the star is measured with respect to the spike, this effect is calibrated.

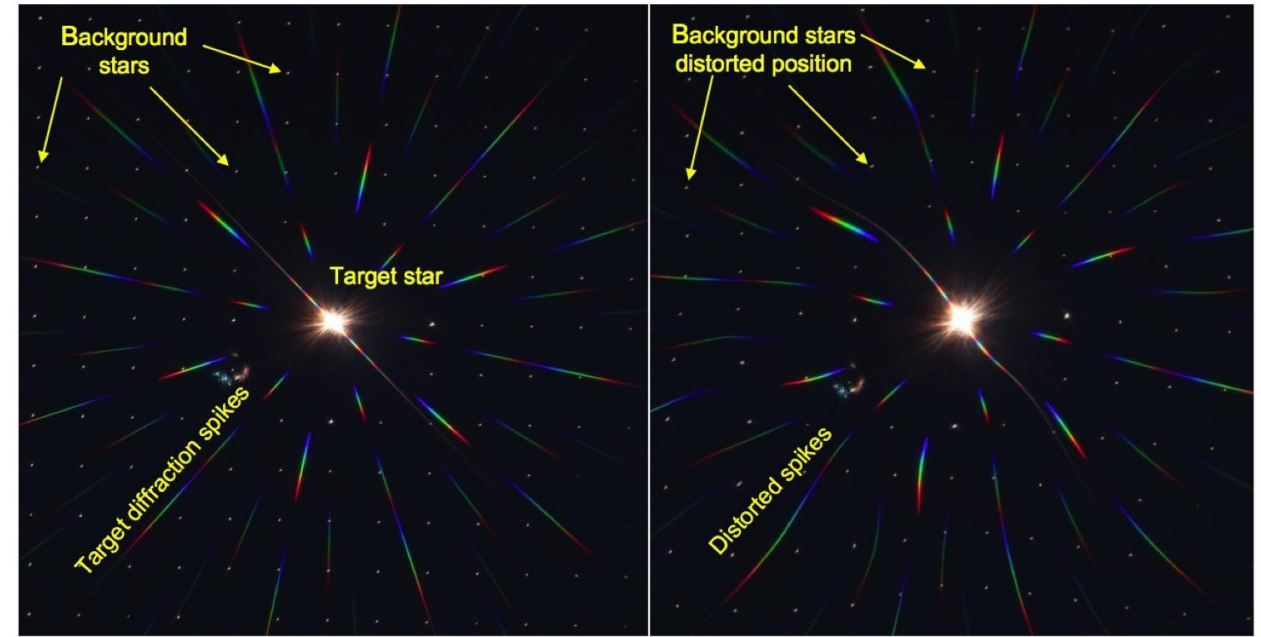

Figure 1.1 Astrometry calibration concept using the diffractive spikes. A system affected by distortions results in errors in the astrometric measurement, which can be calibrated using the diffractive spikes as a reference.

By applying this concept to calibrate dynamic distortions using a DP on a $1.4 \mathrm{~m}$ telescope and additionally rolling the telescope to average down detector effects with a FoV of $0.3 \mathrm{deg}^{2}$, it is possible to achieve $0.2 \mu$ as astrometric accuracy [5]. Since the astrometric signal of the host star is measured with respect to the spikes and not to the star PSF, this technique is compatible with a direct imaging mission. The narrow field around the host star, where the planet is expected, is imaged by a coronagraph, and an astrometric module images the wide field. Therefore, both detection techniques are simultaneously enabled in a single mission. This concept works for a wide range of mission sizes, but the ultimate accuracy strongly depends on the aperture size and the FoV of the telescope.

\section{TESTBED DESIGN AND IMPLEMENTATION}

\subsection{Laboratory overview}

We have designed a testbed to demonstrate the ability of the diffractive pupil to calibrate wide-field imaging distortions and at the same time shows that it is fully compatible with high-contrast imaging. The optical system consists of an imaging system from which the on-axis field is extracted to feed a high-contrast imaging instrument. The remaining offaxis-field is imaged using a wide-field instrument, which delivers the images for astrometry calculation. As a result, our experiment is an end-to-end test bed of simultaneous direct imaging and astrometry mission. Since the experiment is executed in the laboratory, we used an inverted telescope to collimate the light to collimate the light and thereby simulate sources at infinity. 


\subsection{System optical layout}

The system starts with a light source, which has an array of $21 \times 21$ sources arranged on a squared grid separated by $1 \mathrm{~mm}$ each, allowing a field up to $20 \times 20 \mathrm{~mm}$ size. The central source is about four orders of magnitude brighter, and serves as the central star. A super continuum laser manufactured by NKT Photonics, model Super K extreme, is used to feed laser white light to the light source.

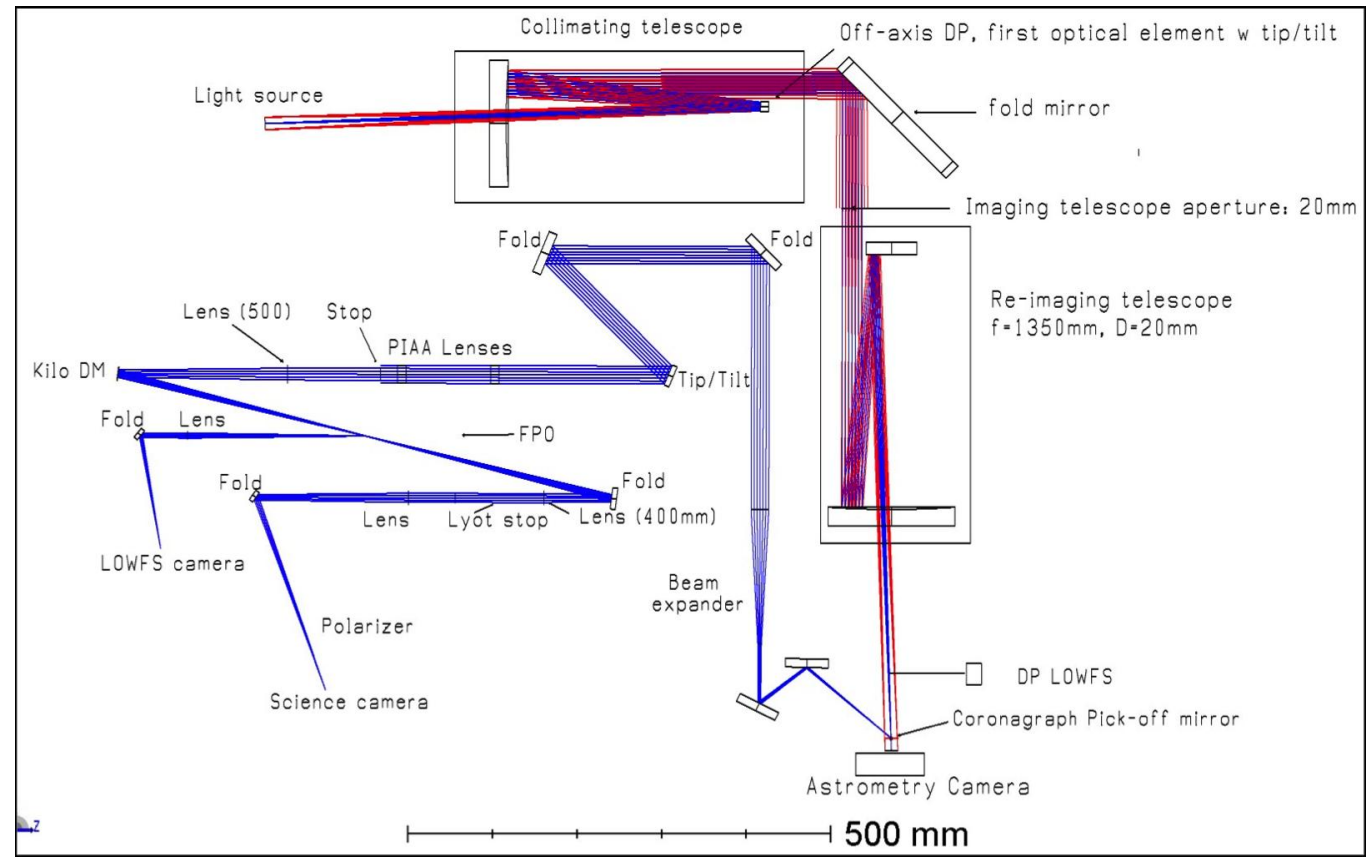

Figure 2.1. Astrometry testbed optical layout showing the light source, the collimating and imaging telescopes. and the coronagraph on the left side.
The

sources are reimaged at infinity using a collimator

telescope, which is an off-the-shelf 6"

Ritchey-Chretien (RC) telescope with a focal length of $\quad 1370 \mathrm{~mm}$ resulting in an $\mathrm{f} / 9$ system. The telescope is reverseilluminated from its focal plane. As a result, its secondary mirror is the first optical surface of the system; hence the DP is imprinted on its surface. We also modified the

telescope to build a hybrid lead-screw and Piezo actuator tip/tilt and focus control for the secondary to stabilize the PSF on a set point. To avoid the diffraction from the telescope spiders, the secondary is stopped down off-axis with an aperture of $16 \mathrm{~mm}$ and an off-axis distance of $20 \mathrm{~mm}$, as shown in Fig 2.1. The red rays corresponds to 19' FoV and the blue is the on-axis field. The collimated off-axis beam that exits the telescope is folded and directed to the imaging telescope, which is another 6" f/9 RC telescope. Before entering its aperture, a $25 \mathrm{~mm}$ diameter polarizer is placed together with $20 \mathrm{~mm}$ stop.

The imaging telescope is the core of the Astrometry instrument, re-imaging the source with a 1-to-1 magnification with an f/68.5 beam..A small pick-off mirror, located $80 \mathrm{~mm}$ upstream of the focal plane, directs the light to a Low Order Wave Front Sensor (LOWFS), which uses a ZWO120MC camera. Tip/tilt measurements of the incoming beam are used to stabilize the image using the active secondary mirror of the collimating telescope for actuation. Table 2.1 summarizes the system parameters.

The Coronagraph instrument starts with a pick-off mirror, shown in Fig.2.3, placed $15 \mathrm{~mm}$ upstream of the focal plane. This mirror has a square $3 \times 3 \mathrm{~mm}$ footprint and extracts the target star FoV to perform high-contrast imaging. The extracted beam remains with $\mathrm{f} / 68.5$ but it is collimated and resized to $18 \mathrm{~mm}$ using a beam expander. After several fold mirrors, an active tip/tilt mirror controls the beam's incidence angle onto the coronagraph; the coronagraph is composed of two Phase Induced Amplitude Apodization (PIAA) lenses
Table 2.1: Astrometry experiment design parameters

\begin{tabular}{|l|c|c|}
\hline Design parameters & Angular & Focal plane \\
\hline Wavelength range & $400-690 \mathrm{~nm}$ & N/A \\
\hline Focal length & $1.37 \mathrm{~m}$ & N/A \\
\hline Aperture D & $0.020 \mathrm{~m}$ & N/A \\
\hline Sampling factor & 3 & N/A \\
\hline Pixels/(L/D) & $6.1 \mathrm{px}$ & $45.1 \mu \mathrm{m}$ \\
\hline Pixel size & $0.165 \lambda / \mathrm{D}$ & $7.4 \mu \mathrm{m}$ \\
\hline Detector size & 2000 & $14.8 \mathrm{~mm}$ \\
\hline HFoV & 18.6 & $7.4 \mathrm{~mm}$ \\
\hline HFoV & $165 \lambda / \mathrm{D}$ & $7.4 \mathrm{~mm}$ \\
\hline Diffractive Pupil & On mirror & Pupil scaled \\
\hline DP spacing $a$ & $120 \mu \mathrm{m}$ & $240 \mu \mathrm{m}$ \\
\hline Dot diameter & $12 \mu \mathrm{m}$ & $24 \mu \mathrm{m}$ \\
\hline DP diameter & $10 \mathrm{~mm}$ & $20 \mathrm{~mm}$ \\
\hline $1^{\text {st }}$ Order location & $55 \lambda / \mathrm{D}$ & Same \\
\hline $2^{\text {nd }}$ Order location & $95 \lambda / \mathrm{D}$ & Same \\
\hline
\end{tabular}


separated by $100 \mathrm{~mm}$, and a $14.5 \mathrm{~mm}$ stop downstream of the PIAA lenses which removes light affected by imperfections beyond the PIAA clear aperture of $16 \mathrm{~mm}$.

The phase apodized beam is focused by a $\mathrm{f}=500 \mathrm{~mm}$ lens. $200 \mathrm{~mm}$ downstream of the lens there is BMC Kilo Deformable Mirror (DM) so that a $9 \mathrm{~mm}$ diameter beam footprint illuminates the surface of the $32 \times 32$ actuator DM, which has a square shape of $9.9 \times 9.9 \mathrm{~mm}$. The DM folds the beam and forms a focus on the Focal

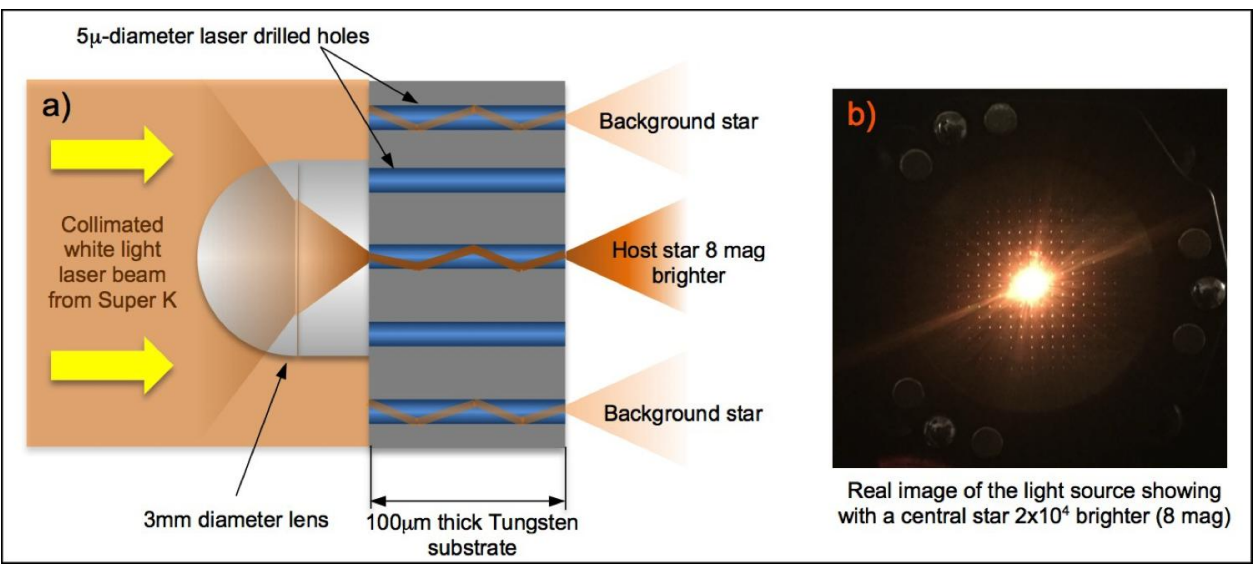

Figure 2.2. The light source and star simulator

Plane Occulter (FPO). The FPO is made of Fused Silica substrate coated with reflective mask on top. The mask has the C-Shape, where the Dark Zone (DZ) is created and the starlight is rejected into a second LOWFS that uses as ZWO178 camera to stabilize in close loop the pre-PIAA tip/tilt mirror.

The light on the DZ continues toward a $400 \mathrm{~mm}$ focal length lens that collimates the light and directs it towards a Lyot Stop that suppresses the remaining rings resulting from residual PIAA apodization on the edges. Afterwards the light goes through a second polarizer before reaching the camera, which is a QSI 543 that has $5.4 \mu \mathrm{m}$ pixels.

\subsection{The light source simulator}

To simulate a star field we created a light source based on a $100 \mu \mathrm{m}$ thick tungsten 1" diameter disc with a grid of $21 \times 21$ laser drilled $5 \mu \mathrm{m}$ holes. The holes, which are equivalent to point sources for the astrometry camera resolution, simulate background stars. The whole plate is illuminated with a collimated white light laser beam generated by the NKT Photonics SuperK Super Continuum laser operating between 400 and $820 \mathrm{~nm}$. On the back of the central hole, a $3 \mathrm{~mm}$ diameter achromatic lens is glued precisely to place its focus on the center of the central $5 \mu \mathrm{m}$ hole, creating a simulated star $\sim 2 \times 10^{4}$ times, or about $8 \mathrm{mag}$, brighter than the background stars. A schematic of the system is shown in Fig. 2.2. The light source is able to roll and align its rotation axis to simulate telescope roll.
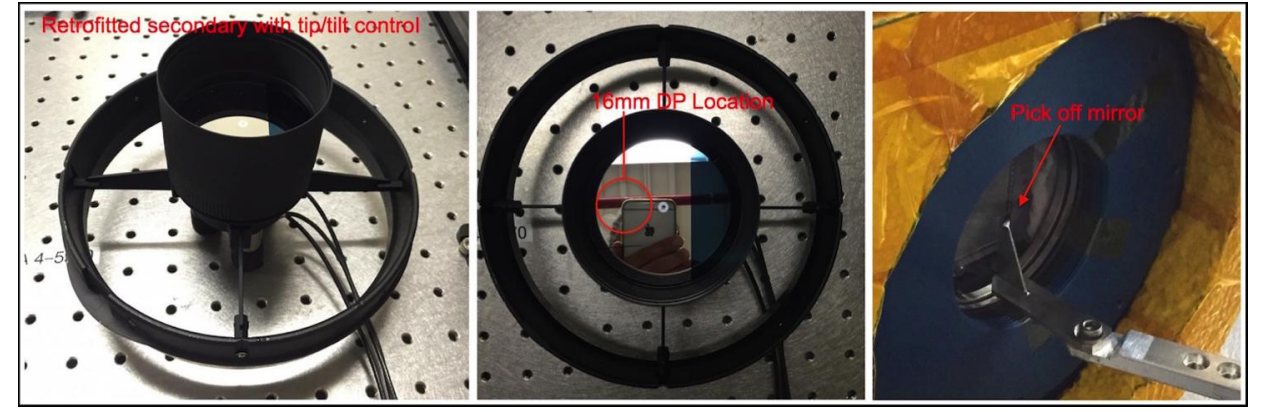

Figure 2.3. Telescope secondary retrofit to add tip/tilt and install the DP on a curved surface. On the right the coronagraph pick-off mirror is shown.

\subsection{Astrometry module design overview}

We have designed the DP to properly sample the Astrometry instrument FoV. There are three top-level requirements that control the DP geometry: the instrument FoV, which controls the dots spacing to ensure equally spaced bright spikes over the FoV, the average brightness of background stars, which defines the dots size in order to maintain a similar surface brightness of the spikes and the stars, and the operational wavelength. This experiment has 19'x19' FoV, with background stars $8 \mathrm{mag}$ dimmer than the target star and it operates in visible light between 400 to $690 \mathrm{~nm}$. The DP location on the telescope secondary is shown in Fig. 2.3.

To calculate the position and size of the dots in the DP we model the hexagonal pattern as a replication of pairs of delta functions over the pupil plane. This can be mathematically represented as, 

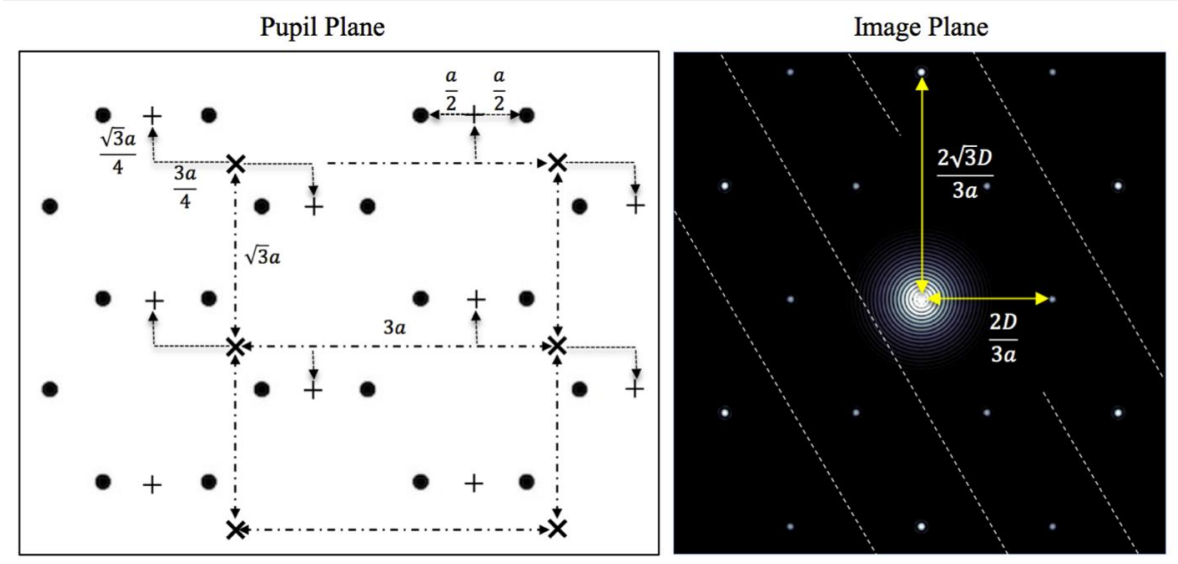

Figure 2.4. On the left, an image of the hexagonal arrangements of dots placed on the pupil is shown. Here, the side of the hexagon is defined as $a$, so the hexagon width is $2 a$ wide. On the right, the resulting image and spots spacing is shown. The dashed lines represent zeros of the cosine modulation that eliminates the spot at D/3a.

$$
g(x, y)=A \operatorname{comb}\left(\frac{x D}{3 a}, \frac{y D}{\sqrt{3} a}\right) *\left[\delta \delta\left(\frac{x D}{\frac{3 a}{4}}, \frac{y D}{\frac{\sqrt{3} a}{4}}\right) * \delta \delta\left(\frac{x D}{a}\right)\right],
$$

where the comb function is a two-dimensional array of delta functions, $\delta \delta$ represents a pair of delta functions, and $A$ represents the scaling factors required to maintain the normalization of delta functions. Note that the axis coordinates $x$ and $y$ have been multiplied by the aperture $D$ to normalize the result to the aperture size. At the image plane the Fourier transform of this grid is obtained as,

$$
G=F_{\xi} F_{\eta}[g(x, y)]=\operatorname{comb}\left(\frac{3 a}{D} \xi, \frac{\sqrt{3} a}{D} \eta\right)\left[\cos \left(\frac{3 \pi a}{2 D} \xi, \frac{\sqrt{3} \pi a}{2 D} \eta\right) \cos \left(\frac{\pi a}{D} \xi\right)\right],
$$

where $\xi$ and $\eta$ represents the transform variables and axes in the image plane. At the image plane we obtain a bi-dimensional comb function with spacing $D / 3 a$ along the $\xi$ axis. However, this grid is modulated by two cosine functions. The first cosine is bi-dimensional and has a period of $4 \mathrm{D} / 3 \mathrm{a}$ along the $\xi$ axis, creating zeros at $\mathrm{D} / 3 \mathrm{a}$ and $\mathrm{D} / \mathrm{a}$ reducing the special frequency of the comb by half allowing deltas only at

$$
\xi=\frac{2}{3} \frac{D}{a}
$$

and at integer multiples of this value. Using the same rationale for the $\eta$ axis, we obtain delta functions at

$$
\eta=\frac{2 \sqrt{3}}{3} \frac{D}{a} \text {. }
$$

Similarly, the deltas are replicated at integer multiples of this value. The spot brightness is modulated by the second cosine of Equation 2, which has a period of $2 D / a$ but does not change the spatial frequency of the grid because it does not have zeros matching the comb period. The geometry described is shown for pupil and image plane in Fig. 2.4.

We designed the system to have spikes starting at

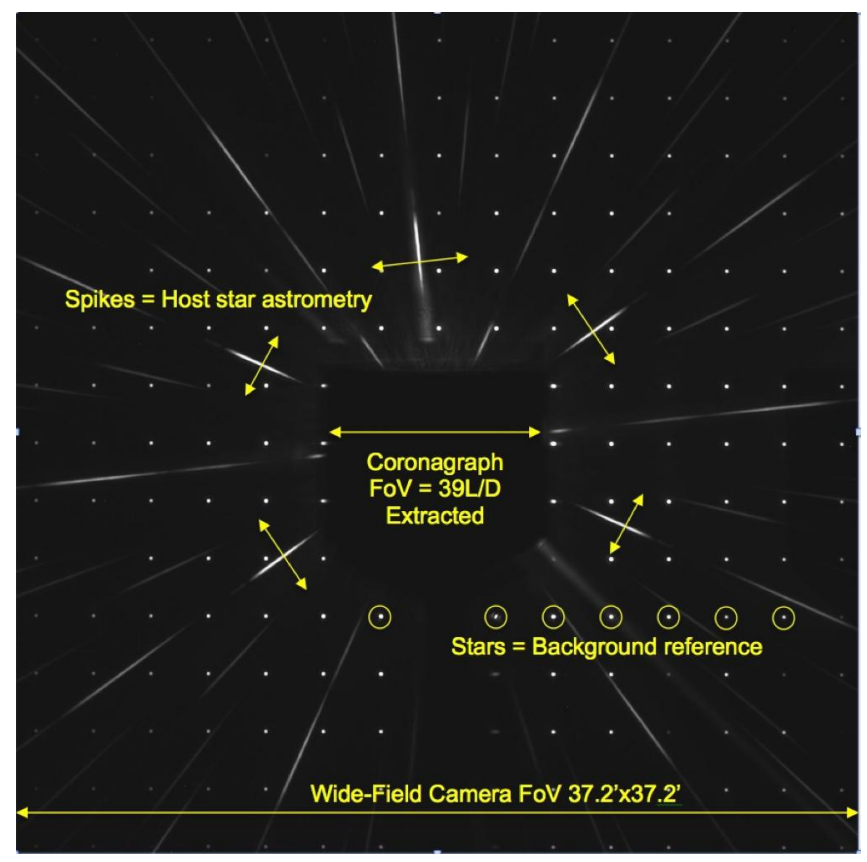

Figure 2.5: Astrometry camera FoV

$55 \lambda / \mathrm{D}$ and $95 \lambda / \mathrm{D}$ for the first and second order respectively. This could be achieved with a DP composed of $12 \mu \mathrm{m}$ dots with a spacing $a=240 \mu \mathrm{m}$ spacing over a $20 \mathrm{~mm}$ pupil. However, we needed to place the DP in the first optical surface, 
which is the secondary of the collimating telescope. As a result, we had to rescale the pupil to its footprint on the secondary, resulting in spacing $a=120 \mu \mathrm{m}$ spacing over a $10 \mathrm{~mm}$ pupil.

Downstrem of the secondary mirror, the primary collimates the beam, which feeds the imaging telescope forming and $\mathrm{f} / 68.5$ beam that creates an image plane that it is sampled by an APOGEE Alta U16000 camera. This camera has $7.4 \mu \mathrm{m}$ pixel size CCD and 16 bits dynamic range. The HFoV is limited to $18.6^{\prime}$ on the camera readout by only reading $2000 x 2000$ px per frame, which is equivalent to $37.2^{\prime}$ or slightly more than half a degree. The resulting image is shown in Fig. 2.5.

\section{ASTROMETRY DATA REDUCTION}

\subsection{Data reduction algorithm}

The data reduction algorithm measures the angular component of the spike positions because accurate radial measurements are prevented by radial smearing of the diffractive features that create the spikes. Nevertheless, the Cartesian X-Y astrometric vector can be obtained by projecting multiple angular displacements into X-Y coordinates.

The algorithm first calculates a reference image, $I_{r e f}$, as the sum of $I_{I}$ and $I_{2}$, and a difference image, $I_{d i f f}$. The next step in the data reduction process is to calculate the angular derivative, which provides a ratio on each pixel to convert the difference image, $I_{\text {diff, }}$, pixel intensity into angular displacement.

To obtain the angular derivative, we first need to compute the Cartesian unitary derivatives of the reference image, $I_{r e f}$. For a pixelated image, we employ discrete differential operators which approximate image derivatives. There are many choices for these operators which vary in accuracy, direction preference, and functionality in the presence of noise, among others. We seek to increase the derivative sensitivity while providing some crude noise smoothing by convolving $I_{r e f}$ with the 3-by-3 isotropic Sobel operator [9]. The x-derivative is shown as follows

$$
\frac{\partial I_{r e f}}{\partial \theta}=\alpha\left(I_{r e f} * f_{x}\right)
$$

where $\alpha$ is a calibration factor and $f_{x}$ is the normalized Sobel operator in the $\mathrm{x}$-direction shown in MATLAB matrix format

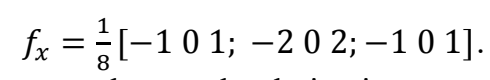

The Cartesian derivative terms are used to compute the angular derivative

$$
\frac{\partial I_{r e f}}{\partial \theta}=-y \frac{\partial I_{r e f}}{\partial x}+x \frac{\partial I_{r e f}}{\partial y},
$$

then the angular displacement is computed by dividing, pixel-to-pixel, the difference image, $I_{\text {diff, }}$ with the angular derivative, given by Equation (7):

$$
\theta_{\text {disp }}=\frac{I_{\text {diff }}}{\frac{\partial I_{r e f}}{\partial \theta}}
$$

At this step the noise, even with some smoothing provided by the Sobel operator, becomes an issue because the distortions to be measured are in the range of $10^{-2}$ to $10^{-5}$ pixels. However, in all other locations of the image where there are no spikes the noise level is orders of magnitude higher. To solve this problem, the SNR of the angular distortion measurement needs to be computed for every pixel. The signal is the value of the angular derivative and the noise is computed as the root sum square of the read-out noise (RON) plus the photon noise,

$$
S N R=\frac{\frac{\partial I_{r e f}}{\partial \theta}}{\text { Noise }}
$$

The angular distortion image is multiplied with the SNR squared, amplifying the values along the spikes and minimizing them on the background,

$$
\theta_{\text {disp_SNR }}=\theta_{\text {disp }} S N R^{2} \text {. }
$$

The image containing the angular distortion is noisy especially along the spikes. A binned version is created to reduce the noise level and the computational power required to process them. This process does not discard useful information because the spikes remain resolved. Then, the angular distortion image is divided by the binned version of $\mathrm{SNR}^{2}$ to 
recover the correct values on the angular distortion image. The pixel value represents the angular distortion for its location in units of pixel size, i.e. a value of 1 represents $7.4 \mu \mathrm{m}$ of angular distortion at that detector location.

The binned angular distortion obtained only contains information available along the spikes. The values for the pixels between the spikes are obtained using a kernel convolution interpolation. The $\mathrm{SNR}^{2}$ and the $\theta_{\text {dist }}$ SNR are binned by a factor of ten to obtain the $\mathrm{SNR}^{2}$ bin and images of $\theta_{\text {dist_SNR_bin }}$ images of $250 \times 250$, which can be interpolated by performing the convolution with a function $g$

$$
\theta_{\text {dist_SNR_bin_interp }}=\left(\theta_{\text {dist_SNR_bin }}\right) * g
$$

where $g$ is a Gaussian kernel defined as

$$
g=e^{-\left(\frac{x^{2}+y^{2}}{2 \sigma^{2}}\right)} .
$$

In Equation 12, $\sigma$ defines the FWHM of the Gaussian kernel. Controlling $\sigma$ will define how aggressive the interpolation is and, therefore, $\sigma$ is set as a parameter in the algorithm that can be adjusted to match the highest spatial frequency distortion expected in the system. Finally, to recover the real values of the angular distortion, it is necessary to divide the $\theta_{\text {dist_SNR_bin }}$ by the $\mathrm{SNR}_{\text {bin_interp }}^{2}$ matrix,

$$
\theta_{\text {dist_real }}=\frac{\operatorname{bin}\left(\theta_{\text {dist }} S N R^{2}\right) * g}{\operatorname{bin}\left(S N R^{2}\right) * g} .
$$

The kernel size is selected to perform the best interpolation between bright spikes at large field angles where most background stars will be found. As we move closer to the center field, the distance between the bright spikes is reduced linearly with the field angle. As a result, the interpolation kernel becomes too large for small fields in the image inducing interpolation errors. To solve this problem, a spatially varying kernel size $g$ can be used to reduce its size, as the interpolation gets closer to the center of the image. This will be studied and implemented for future versions of the laboratory.

\subsection{Algorithm validation}

The algorithm described in the previous section was validated using real data. The test consisted of copying the first epoch real image and shifting it by $0.5 \mathrm{px}$ to the right. Hence, the real distortion to be measured is known, and the algorithm can be evaluated.

Since the spikes do not uniformly sample the distortion and there is no data inside the circle blocked by the occulter, the interpolation result might be biased as a consequence of changing the Gaussian kernel size. If the kernel is too large, it will average valuable high-spatial frequency distortions. A kernel size with FWHM $=55$ was set to provide Nyquist sampling of the spikes in the outer part of the image where most of the stars can be found.

To characterize the effect of the kernel size on the resulting distortion, the algorithm was applied to the spikes and the stars independently for different kernel sizes on a real image and its $0.5 \mathrm{px}$-shifted version. Since there is no real astrometric signal, just an image displacement that is common to both features, the result should be the same for the spikes and the stars, which are perfectly stable in this case.

\subsection{Algorithm optimization}

Using the parameters from the previous section alongside the milestone data, the algorithm is studied by creating a "known distortion" data set. The data set is constructed by taking the first non-dark image of the milestone data as the first epoch in a series of artificially induced sub-pixel shifted epochs. Each subsequent epoch is created by shifting the first epoch by a known amount using an interpolation method with errors smaller than the desired shift amount. The interpolation method employed to create the "known distortion" data set makes use of the Fourier Shift Theorem; the accuracy of this method was verified to the order of 1e-13 mean separation from the desired sub-pixel shift for shifts as small as $0.25 \%$ of a pixel. 
Next, the algorithm is performed on this data set in three separate configurations: first, using the simple derivative approximation of subtracting a onepixel shifted version of $I_{\text {ref }}$ from itself with respect to $X$ and then to Y; second, using convolution with the Sobel operator to compute the derivative; and third, using the Sobel derivative approximation with a calculated calibration factor.

Using the simple derivative approximation in the algorithm, a nonlinear response results from the computed relative and absolute spike distortions in tip and tilt. This response demonstrates that the sensitivity to small shifts by the simple derivative approximation is insufficient for accurate tip/tilt estimates. The calculation of the angular derivative and the $\mathrm{SNR}^{2}$ map are dominated by noise, biasing the interpolation stage of the algorithm and its results. After testing, it was determined that this nonlinear response is most evident for pixel shifts on the order of $1 \%$ of a pixel; the "known distortion" data set used is constructed to demonstrate this effect. The shift between any adjacent images in the set is $0.667 \%$ of a pixel to the right, and $0.5 \%$ of a pixel down, with a total shift of 0.1333 pixels to the right and 0.1 pixels down from the first image to the last.

Fig. 3.1a (top) displays the results of the algorithm on the "known distortion" data set. The relative tip/tilt lines are both curved. The absolute tip/tilt lines show a nonlinear effect over the first few images, and then becomes linear. This is expected because the absolute distortion is computed with respect to the first image, so after three images, the total shift is larger than $1 \%$ of a pixel. The relative distortion is computed from image to image, so the shift stays below $1 \%$ of a pixel, making it more susceptible to nonlinear effects.

Fig. 3.1b (middle) shows the results of the algorithm using the Sobel operator to approximate the derivative. The relative and absolute spike distortions for tilt are coincident; meanwhile the spike distortion lines for tip trend linearly after the first few images. This is a demonstration of the improved sensitivity provided by the Sobel operator derivative approximation with respect to small pixel shifts compared to the previous method. The nonlinear effects are greatly minimized, having been nearly removed

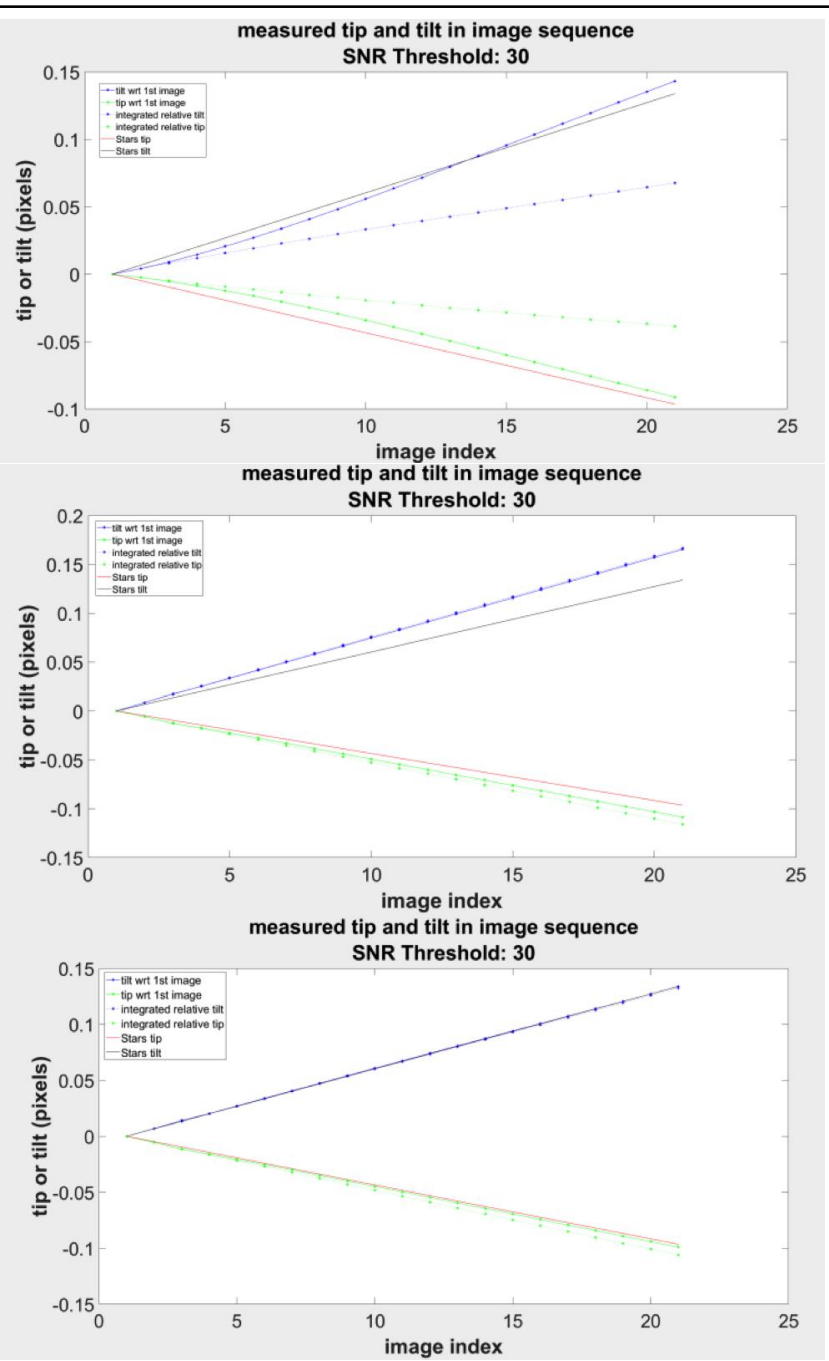

Figure 3.1. The top frame a) shows a comparison of the absolute (solid blue and green), relative (dashed blue and green), and star (black and red) tilt and tip returned by algorithm using one pixelshifted derivative for the artificial data set discussed above. Nonlinear results are clearly observed.

Frame b) shows the same tip and tilts returned by the algorithm for the artificial data set, but with the implementation of the derivative approximation found using convolution with the Sobel operator.

Frame c) show the same tip/tilt returned by the algorithm, following computation and application of the constant multiplicative calibration factors with respect to each shift direction. This brings the final result of the algorithm to desired true tip and tilts added to the artificial data. entirely in the tilt, and only having a nonlinear "bump" at Image Index 3 in the tip before stabilizing to a nearly constant slope.

To make further improvements between the star centroid tip/tilt and spike distortion estimates, calibration factors are computed separately for the star centroids as well as the Cartesian unitary derivatives. The slopes between adjacent epochs along each star and spike curve are computed and the results are averaged together. The computed average functions as a multiplicative calibration factor to correct for non-optimal estimation of the star or spike 
distortions. For the star centroid tip/tilt, the calibration factor is applied to the computed shifts as the last step of the algorithm. This removes any significant bias appearing in Fig. 3.1c (bottom) due to the centroiding algorithm. For the derivatives, the calibration factor is applied to Equation 10. The calibration factor for the derivatives is computed iteratively by repeated application of the algorithm until multiplication of successive calibration factors from each iteration converge to the known distortions beyond a predetermined threshold level. When this has occurred, the respective spike distortion curves become nearly coincident. The results of this process are seen in Fig. 3.1c. It should be noted that the calibration factor must be recomputed if any changes are made to the algorithm (including the geometry of the testbed collecting the data to be analyzed, the binning size, the kernel size, or the SNR threshold).

In all three of these cases, a sensitivity analysis is performed for the SNR threshold value. The results are seen in Fig. 3.2. Each curve represents the results computed at a different SNR threshold in the range of 30-300 in steps of 30, with the direction of increasing value indicated by the arrows on the graph. The absence of an arrow means the data is insensitive to changing the SNR threshold. Figure 3.2a demonstrates a weak sensitivity to the SNR threshold over the range of 30-120 where nonlinear effects from the simple derivative are occurring. As the SNR threshold increases, the noise is reduced over the $\mathrm{SNR}^{2}$ map, resulting in more linear behavior between epochs. The same effect is seen in the tip in Fig. 3.2b (middle); the tilt shows no sensitivity because nonlinearities are not present.

Fig. 3.2c (bottom) shows the results for running the algorithm with constant calibration factors over the range of SNR threshold values. It demonstrates that the calibration factors need to be recomputed as a function of a changing SNR threshold in order to preserve their improvement in the accuracy of the result. This suggests that once an SNR threshold is chosen and the calibration factors are computed, the threshold value should not be changed to calculate consistent results.

The above analysis suggests equipping the algorithm with a more sensitive, noise smoothing derivative approximation such as the Sobel operator coupled with multiplicative calibration factors is capable of returning the known distortions in artificially created data.

Further optimization of the algorithm is being considered. This includes masking the stars/spikes to calculate their distortion estimates separately. If done carefully, this should help to alleviate the need for the multiplicative calibration factors, as the presence of the stars in the spike calculations provides a portion of the bias that the calibration factor is removing. Further

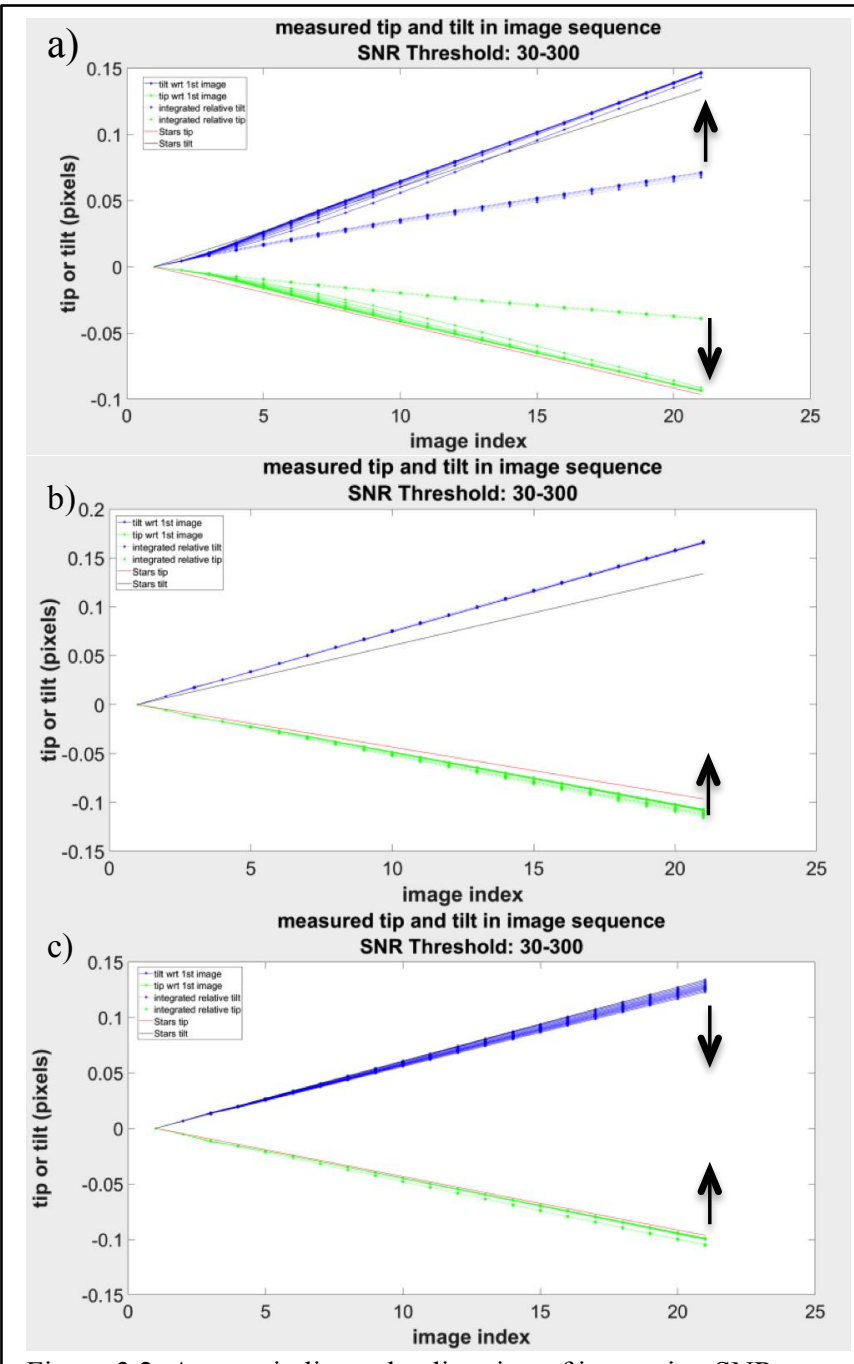

Figure 3.2. Arrows indicate the direction of increasing SNR threshold. Top frame a) displays the SNR threshold sensitivity analysis performed using neither of the optimization methods discussed above. Sensitivity is seen over the range of threshold values of 30-120 in the regions where a nonlinear behavior is also observed.

Frame b) displays the SNR threshold sensitivity analysis when using Sobel derivative approximation. Almost no sensitivity is observed in the tilt direction, as the results are very nearly linear. Sensitivity is noted in the tip direction because of the slight nonlinearity at the third image index.

Frame c) displays the SNR threshold sensitivity analysis when using both the Sobel derivative approximation and multiplicative calibration factors. Sensitivity to the SNR threshold is noted over the entire range tested. This shows that the multiplicative calibration factor is sensitive to the threshold value for which the algorithm is run to compute it (in this case the lowest value, 30). 
optimization can include creating an adaptively sized interpolation kernel. This includes a parameter space of where in an epoch the kernel should change size, and to what size it should change to while interpolating.

\section{RESULTS}

The experiments and measurements performed using this testbed are planned to meet two TDEM milestones. Completion of these milestones is to be documented in a report and reviewed by the Exoplanet Exploration Program.

\subsection{Milestones definition}

This project has two milestones, which read as follows:

Milestone \#1 definition:

Broadband medium fidelity imaging astrometry demonstration

Demonstrate $2.4 \times 10^{-4} \lambda D$ astrometric accuracy per axis performing a null result test. The laboratory work will be carried out in broadband spectrum covering wavelengths from 450 to 650nm using an aperture pupil (D) equal or larger than $16 \mathrm{~mm}$.

The "angular separations" are defined in term of the source wavelength $\lambda$, and the diameter $D$ of the aperture on the DP, which is the pupil-defining element of the imaging astrometry camera. For this milestone, a DP simulates the telescope primary and pupil and it will illuminated by an array of broadband point sources forming f/25 to f/50 beams when they reach the pupil.

\section{Milestone \#2 definition:}

Broadband medium fidelity simultaneous imaging astrometry and high-contrast imaging Demonstration of milestone $\# 1$, and performing high-contrast imaging achieving $5 \times 10^{7}$ raw contrast between 1.6 and $6 \lambda / D$ by a single instrument, which shares the optical path, from the source to the coronagraphic and astrometry FoV separation. The ability of achieving $5 \times 10^{7}$ raw contrast will be considered as proof of no contamination of the inner working angle (IWA).

\subsection{Astrometry results}

The astrometry experiment was performed first, however the whole optical train was already installed. This is important because the coronagraph pic-off mirror edges diffract a small amount of light into the wide-field image. The astrometry results were based on a Null test defined as follows.

\subsubsection{Null test definition}

The goal of the Null Test is find the level of accuracy that the system will be able to measure no motion of the system. The residual error of the null test quantifies the ability of the system to perform relative measurements necessary to detect and characterize planets using imaging stellar astrometry at different epochs in time.

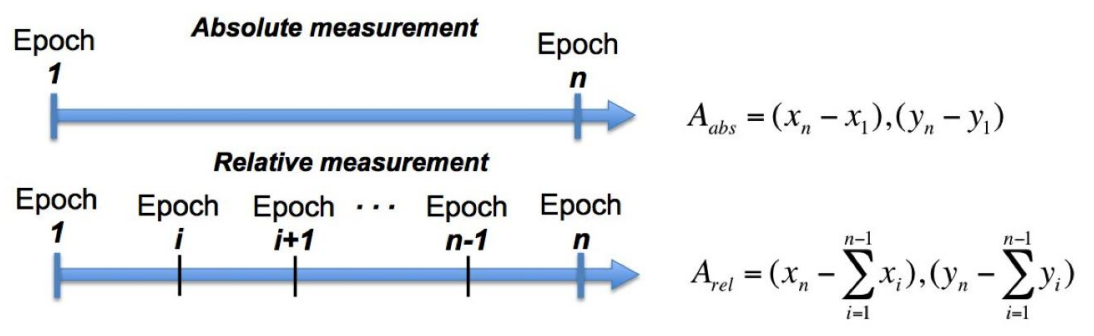

Figure 4.1: Relative vs. absolute measurement

Our Null Test success criterion is based on the comparison of relative incremental measurements, equally spaced and absolute measurement between two astrometry epochs as shown in Fig. 4.1. To successfully meet the milestone, the difference between the absolute, 2-point measurement and the sum of $n$ incremental measurements between those point is smaller than $2.4 \times 10^{-4}$ $\lambda D$, at least 10 epochs are required

over 40 hours.

We performed three runs, each $48 \mathrm{hrs}$ long, with 12 epochs, one every $4 \mathrm{hrs}$, and used five images per epoch. The bench was stabilized to $22.5^{\circ} \mathrm{C}+/-20 \mathrm{mK}$ over $48 \mathrm{hrs}$. The LOWFS stabilized the PSF down to $0.14 \mathrm{px}$ and $0.11 \mathrm{px}$ RMS on the ZWO camera that has $3.4 \mu \mathrm{m}$ pixel size, which is equivalent to $1.06 \times 10^{-2} \lambda / D$ and $8.34 \times 10^{-3} \lambda / D$ stability for the $\mathrm{X}$ and $\mathrm{Y}$ axes respectively in the astrometry camera. However, maintaining such a stable positioning of the image required DM strokes of up to $4.65 \mathrm{~V}$ and $1.27 \mathrm{~V} \mathrm{PV}$, equivalent to a 1.92 " tip and 0.54 " tilt of the secondary mirror, which would 
cause significant beam walk and alter the star positions. Our stabilized performance proves the resilience of this technique to beam walk and optics deformation..

\begin{tabular}{|c|c|c|c|c|c|}
\hline Table 4.1: Milestone \#1 & Null test RMS $(\lambda / \mathrm{D})$ & Milestone \#1 $(\lambda / \mathrm{D})$ & \multicolumn{2}{c|}{ Stars only null test $(\lambda / \mathrm{D})$} \\
\cline { 2 - 6 } & $\mathrm{X}$-axis & Y-axis & 1 -axis & X-axis & Y-axis \\
\hline TDEM result & $\mathbf{2 . 3 8 e - 0 5}$ & $\mathbf{4 . 0 0 e - 5}$ & $2.4 \mathrm{e}-4$ & $7.09 \mathrm{e}-04$ & $1.65 \mathrm{e}-03$ \\
\hline Equivalent for $\mathrm{D}=2.4 \mathrm{~m}$ & $1.0 \mu \mathrm{as}$ & $1.7 \mu \mathrm{as}$ & $10.0 \mu \mathrm{as}$ & $>23.4 \mu \mathrm{as}$ & $>39.0 \mu \mathrm{as}$ \\
\hline Equivalent for $\mathrm{D}=4.0 \mathrm{~m}$ & $0.6 \mu \mathrm{as}$ & $1.0 \mu \mathrm{as}$ & $6.2 \mu \mathrm{as}$ & $>5.79 \mu \mathrm{as}$ & $>9.65 \mu \mathrm{as}$ \\
\hline
\end{tabular}

The results are shown in Table 4.1. We were able to achieve $2.38 \times 10^{-5} \lambda / \mathrm{D}$, which is 10 times better that the milestone. Fig. 4.2 shows the residual error between cumulative and absolute measurements used to compute the values in Table 4.1 .

To compare the performance of the diffractive pupil with a traditional star centroiding approach, we applied the null test to the background star field. The results are shown on the rightmost columns on Table 4.1, and on the plot on the right of

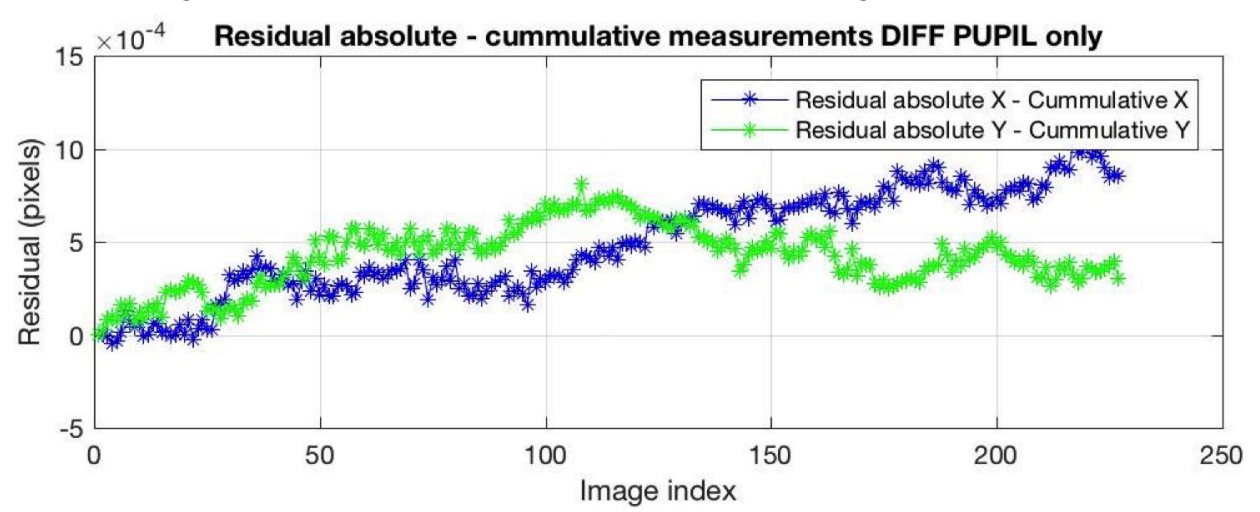

Figure 4.2: Residual error between the cumulative and absolute measurements for each image.

Fig. 4.3. The RMS error of the null test is more than 20 times larger than using the spikes. We also compared the null test error obtained when the stars are used as reference with the beam walk in the optical system, which is caused by tip/tilt motions of the secondary of the collimating telescope required to stabilize the images as residual thermal drifts deforms the optical system. The correlation between the signals is the result of distortion caused by beam walk, and the resilience of the spikes to the same perturbation demonstrates the ability of the diffractive pupil to correct those errors.

The accuracies demonstrated of $2.38 \times 10^{-5} \lambda / \mathrm{D}$ would enable a $2.4 \mathrm{~m}$ diameter telescope to achieve $1 \mu$ as
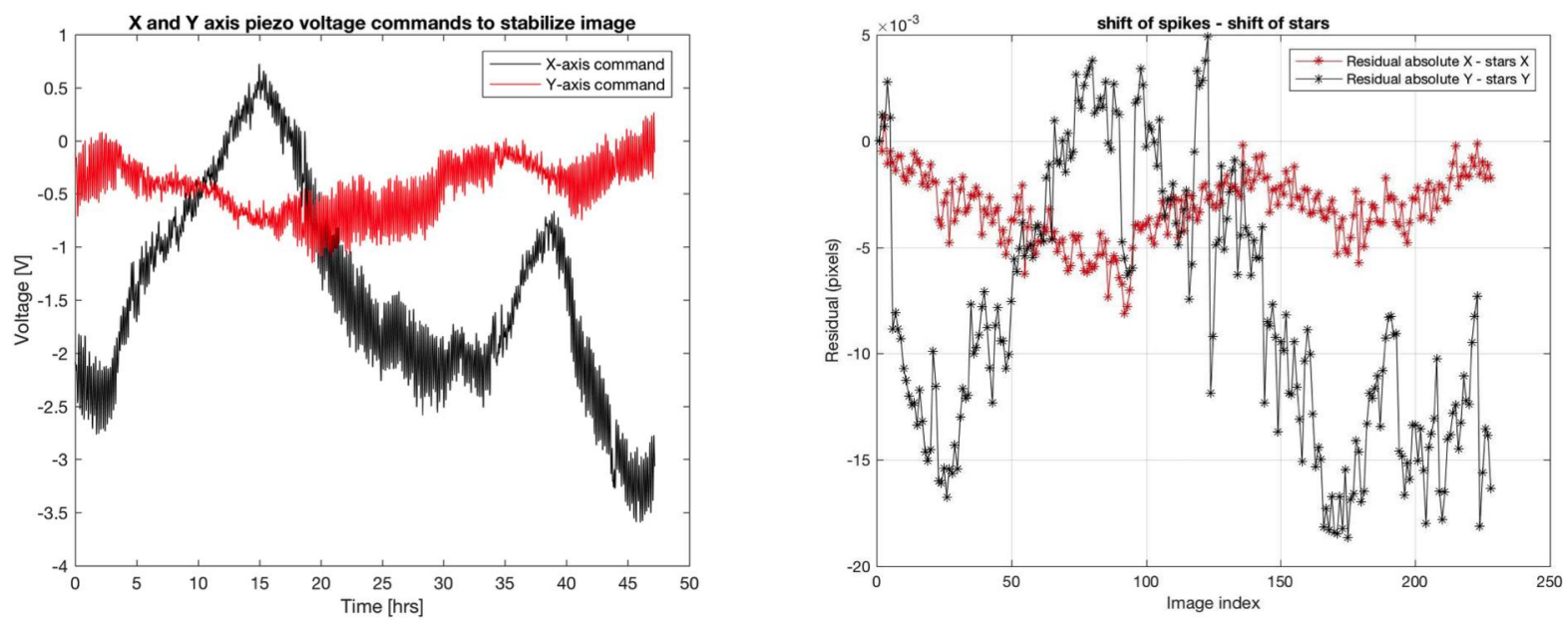

Figure 4.3: Comparison of the control signal applied to the collimating telescope tip/tilt mirror (right) and the star's only positing error (left). The two plots cover the same period of time. The correlation between tip/tilt and error is caused by beam walk that biases the stars position. 
accuracy, and hence detect and measure masses of earth-like planets around sun-like stars within $3 \mathrm{pc}$. In contrast, the null test using the stars shows an accuracy of $23.4 \mu$ as for a $2.4 \mathrm{~m}$ diameter telescope, which is consistent with the best performance reported by Hubble using special scanning techniques. Also, if we extrapolate this to a $4 \mathrm{~m}$ telescope such as HabEx, our astrometry null test would achieve sub-1 $\mu$ as accuracy, whereas the stars only null test would only scale to $\sim 6 \mu$ as accuracy, insufficient to find and characterize earth-like planets.

\subsection{Direct imaging results}

The technology demonstration behind milestone \#2 is to prove that high-contrast imaging techniques, including coronagraphs and wavefront control, remain operational and unaffected by the addition of a DP upstream of the coronagraph. Also, it allows testing of a full end-to-end system demonstration. The system layout and the coronagraph described in section 2.3 were used to perform the following tests.

Monochromatic laser light at $655 \mathrm{~nm}$ was fed through the same optical path shown in the optical layout. The pick-off mirror extracts the high-contrast imaging FoV and feeds the coronagraph. For each run we use the following procedure:

- Align the PSF using the pre-PIAA tip/tilt mirror to remove any residual off-axis terms

- Calibrate plate scale sending sine waves on the DM

- Insert the FPO rejecting star light to the PIAA LOWFS

- Image the rejected star light onto the LOWFS camera, then turn on the PID control loop

- Increase laser power to create visible speckle

- Execute speckle nulling to create the DZ

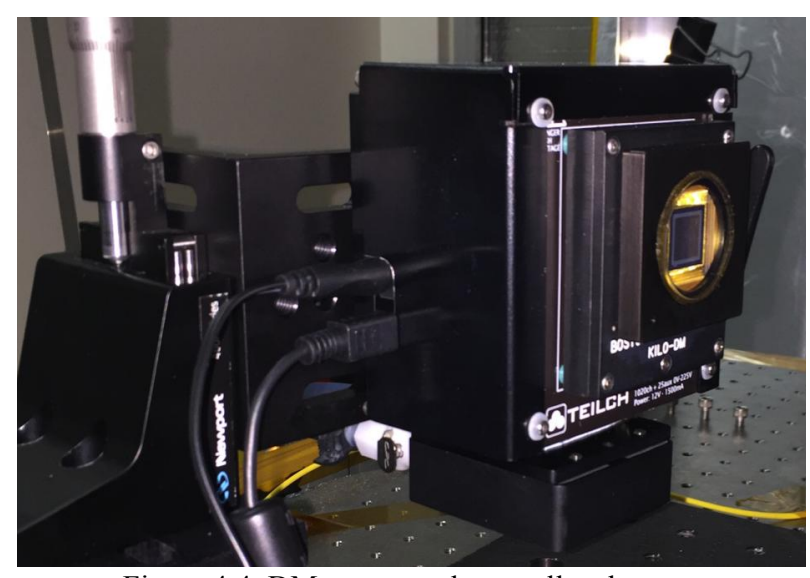

Figure 4.4: DM mount and controller shown installed at the bench

The residual rejected PSF jitter for the $\mathrm{X}$ and $\mathrm{Y}$-axis is $1.41 \times 10^{-2} \lambda / \mathrm{D}$ and $5.97 \times 10^{-3} \lambda / \mathrm{D}$ RMS after stabilization. The control loop was running slow at only $1 \mathrm{~Hz}$ to control drifts, but not to suppress vibrations. The bench would be stabilized to $+/-20 \mathrm{mK}$ during the run. A typical run would last 2 days starting from a flat DM. The miniaturized DM controller was used to control the mirror with very good results. A unique feature on this bench is that the DM is directly attached to the controller, which is supported by translation and rotation stages. Power and control are supplied using a $12 \mathrm{~V}$ jack and a USB3 cable for data. The system generates a maximum of $8 \mathrm{~W}$ of heat, which is removed using a heat exchanger below the controller shown in Fig. 4.4.
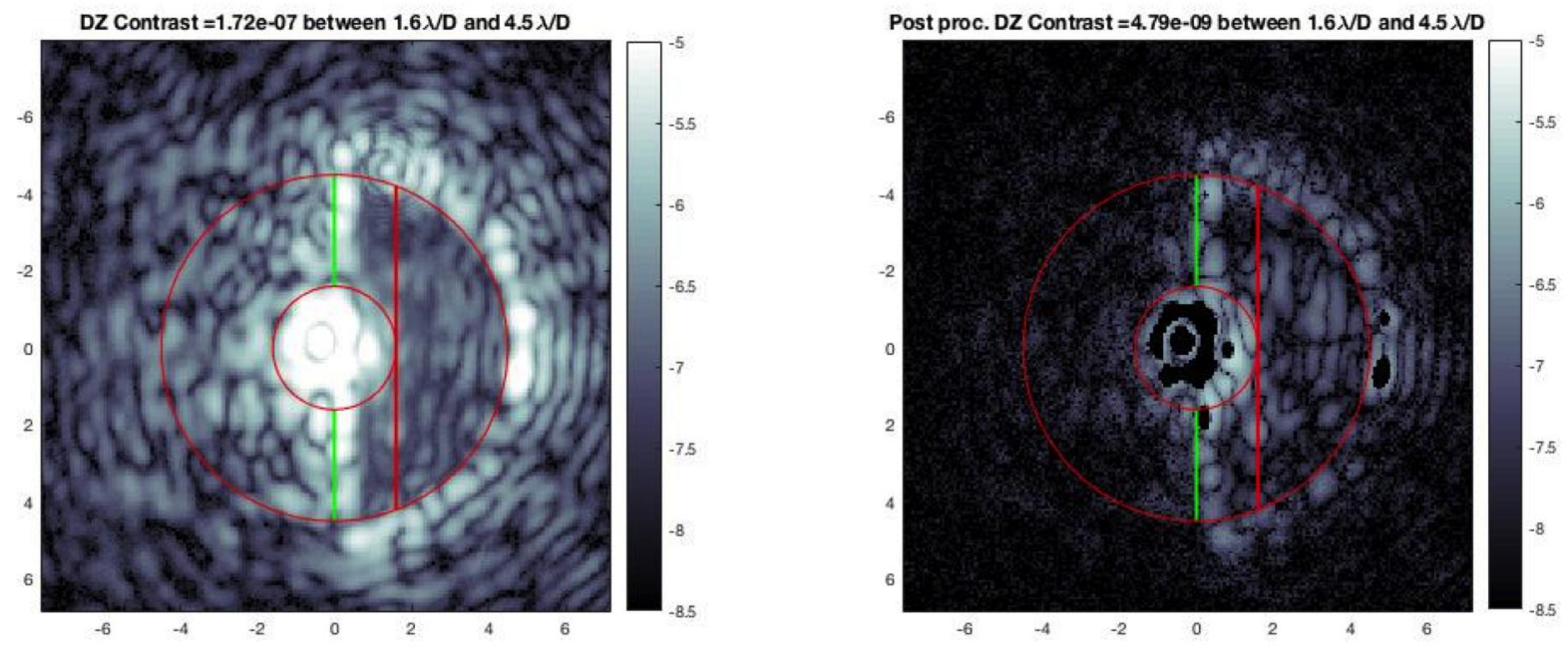

Figure 4.5: DM high contrast imaging results, raw on the left, with post processing on the right. 
Typical contrast consistently achieved during the runs was in the order of $2 \times 10^{-7}$ from 1.6 to $4.5 \lambda / \mathrm{D}$, which is more than a factor of 2 than the milestone contrast requirement of 5.0 $\times 10^{-7}$. The outer working angle is limited by the size of the $\mathrm{C}$-shape of the occulter but is not a fundamental problem of the wavefront control algorithm or contamination cause by the DP. The example shown in Fig. 4.5 (left) is the best contrast in set 3 , with $1.72 \times 10^{-7}$ contrast from 1.6 to $4.5 \lambda / \mathrm{D}$. At this contrast level, there is no indication of an impact from the DP and it was difficult to get a better contrast due to effects from the PIAA lenses, mainly reflections between lenses flat surfaces.

To explore the possibility of IWA contamination further, we performed a basic post processing of the images. After the best contrast was achieved, the amplitude of the speckle nulling correction was dialed down to zero to maintain the configuration's stability. The contrast evolution versus time is shown in Fig. 4.6. A set of 39 iterations was recorded and the most stable part of the set, which was during the first 25 images, and it was used to average a reference frame. This

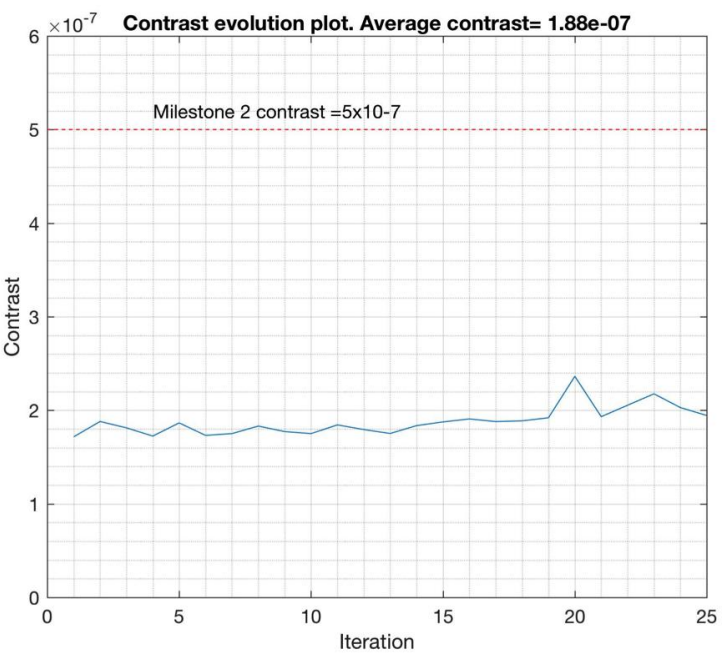

Figure 4.6: Contrast stability test frame was subtracted to the best contrast image in the set resulting in DZ with an average contrast of $4.79 \times 10^{-9}$ (Fig 4.5 right) in which we could verify there is no DP impact. Note that this simple post processing approach allowed us to gain a factor of 35 in contrast, which could be used if the planet is moving and it will not be affected by the subtraction operation.

\section{CONCLUSIONS}

Mass determination of exoplanets is necessary to characterize exoplanets and assess their habitability. However, this measurement remains elusive due to accuracy limitations of current instrumentation. Radial Velocity (RV) measurements have been used as the workhorse to measure exoplanet masses, but this technique can only constrain the planet mass because the system inclination is unknown. In some cases RV measurements can be obtained from known transiting exoplanets, and in such cases the mass can be precisely determined, but it is a small fraction of the exoplanet population. Astrometry offers a unique opportunity to detect and measure exoplanet masses for any system inclination.

In this paper we have described the combined direct imaging and astrometry testbed developed as part of a NASA's TDEM-13 funded effort to advance the diffractive pupil technology. As part of this effort, together with University of Arizona we have developed techniques to imprint the diffractive pupil in curved mirrors, which are utilized in our testbed, and prepared the technology for scalable capabilities to cover large mirrors.

We have successfully met the milestones defined for this effort by demonstrating $2.38 \times 10^{-5} \lambda / \mathrm{D}$ astrometric accuracy per axis performing a null result test. This result is 10 times better than milestone \#1 that requires $2.4 \times 10^{-4} \lambda / \mathrm{D}$ astrometric accuracy. Also we demonstrated the compatibility with high-contrast imaging, achieving $1.72 \times 10^{-7}$ raw contrast which is almost three times better than milestone \#2 requirement two of $5 \times 10^{7}$ raw contrast between 1.6 and $6 \lambda / \mathrm{D}$ by a single instrument that shares the optical path, from the source to the coronagraphic and astrometry FoV split.

We consider this a high-fidelity demonstration of the technique because $2.38 \times 10^{-5} \lambda / \mathrm{D}$ astrometric accuracy is equivalent to $1 \mu$ as on a $2.4 \mathrm{~m}$ telescope operating in the visible and sub- $\mu$ as accuracy on larger flagship missions such as HabEx or LUVOIR. Such accuracy would enable detection and mass measurements of earth-like planets around sun-like stars within 10pc while simultaneously performing high-contrast imaging of the same target.

\section{ACKNOWLEDGMENTS}

This project is funded by a NASA's Technology Development for Exoplanet Missions program grant awarded on 2013. We acknowledge the support of JPL's Exoplanet Exploration Program, in particular Nick Sigler and Brendan Crill. 


\section{REFERENCES}

[1] Papaloizou, J., Terquem, C., "Dynamical relxation and massive extrasolar planets," MNRAS, 325, 221-230. (2001).

[2] Lunine, J.I., O'Brien, D. P., Raymond, S. N., Morbidelli, A., Quinn, T., Graps, A., "Dynamical models of terrestrial planet formation," arXiv0906.4369L, (2008).

[3] Shao, M., Catanzarite, J., Pan, X., "The Synergy of Direct Imaging and Astrometry for Orbit Determination of ExoEarths," Apj, 720, 357-367. (2010).

[4] Guyon, O., et al., "Simultaneous Exoplanet Characterization and Deep Wide-field Imaging with a Diffractive Pupil Telescope," ApJ, 767, 11. (2013).

[5] Guyon, O et al., "High precision astrometry with a diffractive pupil telescope," ApJS, 200, 11. (2012).

[6] Trippe, et al., "High-precision astrometry with MICADO at the European Extremely Large Telescope," MNRAS, 402, 1126-1140. (2010).

[7] Marois, C., et al., "Accurate Astrometry and Photometry of Saturated and Coronagraphic Point Spread Functions," ApJ, 647, 1, 612-619. (2006).

[8] Sivaramakrishnan, A., Oppenheimer, B., "Astrometry and Photometry with Coronagraphs," ApJ, 647, 1, 620-629. (2006).

[9] Sobel, I., \& Feldman, G., “A $3 \times 3$ isotropic gradient operator for image processing," Presented at a talk at the Stanford Artificial Project. (1968).

[10]Bendek, E., et al., "Laboratory demonstration of astrometric compensation using a diffractive pupil," accepted PASP, October 1st (2013).

[11]Bendek, et al., "Laboratory demonstration of coronagraphic imaging with a diffractive pupil," PASP, 125, 924, 204212. (2013). 